\title{
The utility of PET imaging in the diagnosis and management of psychosis: a brief review
}

\author{
Grant Rigney $^{1} \cdot$ Cyrus Ayubcha ${ }^{2} \cdot$ Thomas J. Werner $^{3} \cdot$ Abass Alavi $^{3} \cdot$ Mona-Elisabeth Revheim $^{4,5}$ (D)
}

Received: 22 July 2021 / Accepted: 20 September 2021 / Published online: 2 October 2021

(c) The Author(s) 2021

\begin{abstract}
Purpose Advances in the pathophysiological characterization of psychosis has led to a newfound role of biomarkers in diagnostic and prognostic contexts. Further, advances in the accuracy and sensitivity of nuclear medicine imaging techniques, and specifically positron emission tomography (PET), have improved the ability to diagnose and manage individuals experiencing first-episode psychosis or those at greater risk for developing psychosis.

Methods Literature searches were performed in PubMed, Google Scholar, and Web of Science to identify papers related to the use of PET imaging in the diagnosis or management of psychosis. Search terms used included "positron emission tomography", "PET imaging", "psychosis", "disorders of psychosis", "schizophrenia", "biomarkers", "diagnostic biomarkers", "prognostic biomarker", "monitoring biomarker", "outcome biomarker", and "predictive biomarker."

Results Studies included fell into three categories: those examining microglia, those studying dopamine synthesis capacity, and those examining acetylcholine receptor activity. Microglial imaging has been shown to be ineffective in all patients with psychosis, but some believe it shows promise in a subset of patients with psychosis, although no defining characteristics of said subset have been postulated. Studies of dopamine synthesis capacity suggest that presynaptic dopamine is reliably elevated in patients with psychosis, but levels of dopamine active transporter are not. Further, positron emission tomography (PET) with [18F]fluoro-L-dihydroxyphenylalanine $\left(\left[{ }^{18} \mathrm{~F}\right] \mathrm{FDOPA}\right)-\mathrm{PET}$ has been recently used successfully as a predictive biomarker of dopaminergic treatment response, although more work is needed to validate such findings. Finally, existing studies have also documented lower levels of binding to the $\alpha 7$ nicotinic cholinergic receptor $(\alpha 7$-nAChR $)$ via $\left[{ }^{18} \mathrm{~F}\right]$-ASEM PET in patients with psychosis, however there is a dearth of prospective, randomized studies evaluating the efficacy of $\left[{ }^{18} \mathrm{~F}\right]-\mathrm{ASEM}$ as a diagnostic or monitoring biomarker of any kind.

Conclusion Molecular imaging has become a useful tool in the diagnosis and management of psychosis. Further work must be done to improve the comparative prognostic value and diagnostic accuracy of different radiotracers.
\end{abstract}

Keywords Psychosis $\cdot$ PET imaging $\cdot$ FDOPA $\cdot$ Microglia $\cdot$ First-episode psychosis

\section{Introduction}

Mona-Elisabeth Revheim

mona.elisabeth.revheim@ous-hf.no

1 Department of Psychiatry, University of Oxford, Oxford, England, UK

2 Harvard Medical School, Boston, MA, USA

3 Department of Radiology, Hospital of the University of Pennsylvania, Philadelphia, PA, USA

4 Division of Radiology and Nuclear Medicine, Oslo University Hospital, Oslo, Norway

5 Institute of Clinical Medicine, Faculty of Medicine, University of Oslo, Oslo, Norway

\section{Defining biomarkers}

A biomarker is a "characteristic that is objectively measured and evaluated as an indicator of a normal biological process, pathologic process, or response to a therapeutic intervention" [1]. Imaging biomarkers are considered either diagnostic or outcome makers. As their name suggests, diagnostic markers can support the diagnosis of a condition. Of these outcome markers their utility can be considered in the context of monitoring, prognostication, and prediction. Monitoring biomarkers track the course of disease (e.g., tau burden in Alzheimer's disease). Prognostic biomarkers suggest the 
nature of prospective disease progression without therapeutic intervention; whereas, predictive biomarkers project patients' potential therapeutic response [2].

\section{Psychosis and the potential role of molecular imaging}

While not possessing an exact clinical definition, psychosis is characterized by common symptoms of delusions, hallucinations, and thought disorders [3]. Several different psychiatric illnesses in the Diagnostic and Statistical Manual of Mental Disorders, fifth edition include psychosis as a symptom, including schizophrenia, schizophreniform disorder, schizoaffective disorders, brief psychotic disorders, delusional disorder, bipolar disorder, and more [4]. For the purpose of this review, the term psychosis will be used generally to include imaging of an individual who is actively experiencing symptoms of psychosis, regardless of the root disorder from which it stems.

The global burden of psychosis is substantial, with the incidence of all psychotic disorders being 26.6 diagnoses per 100,000 person-years [5]. The current gold standard for diagnosing a psychotic disorder is reliant upon symptom recognition and expert opinion [6]; therefore, diagnostic classification and validity across the profession are essential. Nevertheless, there remains uncertainty in diagnosing psychosis and its various forms as a result of this subjective criteria; emerging evidence suggests that quantitative biomarkers may diagnose and distinguish between forms of psychosis [7]. Thus, quantitative imaging of such biomarkers has presented great promise as a diagnostic tool for psychosis.

Approximately one third of patients with schizophrenia are non-responsive to first-line antipsychotic therapies [8]. The continuance of antipsychotic medications for such patients can be medically, socially, emotionally, and financially detrimental, making it advantageous to identify which patients can respond to first-line therapies. Innovative efforts have developed ligands used in positron emission tomography (PET) imaging that can not only identify individuals with psychosis but also determine who will respond to antipsychotic treatment. Such markers offer the possibility of improving treatments, increasing diagnostic accuracy, and improving our biological understanding of psychosis.

Several mechanisms have been proposed to explain psychosis where the involvement of relevant biomarkers in the respective theories has greatly expanded the role of PET imaging biomarkers for psychosis. Previous reviews have examined either (1) all PET imaging biomarkers being studied for their application to psychosis [9] or (2) a restricted subset of PET imaging biomarkers procured for a particular argument [10]. This review utilizes an extensive base of scientific and clinical studies to examine all PET biomarkers with respect not only to their scientific value in characterizing psychosis but also their immediate clinical relevance. Importantly, some PET imaging biomarkers (e.g., markers of metabolism and hypofrontality) will be excluded, as a dearth of existing literature suggests that their immediate clinical relevance in the diagnosis or management of psychosis is limited. The objective of this scoping review was to critically evaluate the potential utility of PET imaging in the management of psychosis.

\section{Methods}

Literature searchers were performed in PubMed, Google Scholar, and Web of Science to identify studies reporting on the use of PET imaging in the treatment or diagnosis of people with psychosis. Searchers were performed using the words "positron emission tomography", "PET imaging", "psychosis", "disorders of psychosis", "schizophrenia", "biomarkers", "diagnostic biomarkers", "prognostic biomarker", "monitoring biomarker", "outcome biomarker", and "predictive biomarker" from journal inception to current for each journal. References of studies used to inform this review were also examined for additional relevant studies not indexed by the literature searches. Studies were not included in this review if they did not contain information specifically on PET imaging of individuals with psychosis, or if they did not contain information on the use of diagnostic or outcome biomarkers in the management of psychosis. As this was not a systematic review or meta-analysis, no statistical analysis was performed.

\section{PET biomarkers of psychosis}

\section{Diagnostic biomarkers}

\section{Neuroinflammation and TSPO-PET imaging}

Increased microglial activation and subsequent neuroinflammation is known to be associated with schizophrenia and other related disorders of psychosis [11, 12]. Various PET studies using $\left[{ }^{11} \mathrm{C}\right]-(\mathrm{R})-\mathrm{PK} 11195$ and $\left[{ }^{11} \mathrm{C}\right] \mathrm{PBR} 28$ have found increased radiotracer binding to the translocator protein (TSPO) in patients with chronic psychosis, first-episode psychosis, and those at high-risk for developing psychosis [13-15], although other tracers have also been used. Thus, imaging neuroinflammation via TSPO binding is believed to be reliably elevated in patients with schizophrenia and other forms of psychosis. However, as many TSPO ligands are elevated in patients with diseases other than schizophrenia, there is considerable difficulty concluding that TSPO ligands can be considered a necessary and sufficient 
diagnostic biomarker. Rather, many, including those in the studies describe herein, have used them alongside clinical judgement to monitor the biochemical milieu in those with schizophrenia.

While there are many studies supporting the utility of TSPO-PET, recent research has added nuance to the role TSPO-PET can play in evaluating patients with psychosis. A meta-analysis examining TSPO-PET (using $\left[{ }^{18} \mathrm{~F}\right]-\mathrm{PBR} 111$, $\left[{ }^{11} \mathrm{C}\right]-\mathrm{PK} 11195, \quad\left[{ }^{11} \mathrm{C}\right]-\mathrm{PBR} 28, \quad\left[{ }^{11} \mathrm{C}\right]-\mathrm{DPA}-713$, $\left[{ }^{18} \mathrm{~F}\right]$-FEPPA, and $\left[{ }^{11} \mathrm{C}\right]$-DAA1106) in 190 patients with psychosis and 200 healthy controls reported that the binding potential $\left(K_{i}^{\text {cer }}\right)$ of TSPO-PET was sufficiently sensitive to detect an elevation in TSPO levels in patients with psychosis, but the volume of distribution $\left(V_{T}\right.$, or the ratio of the concentration of the ligand in the target tissue to that in the plasma) was not sufficiently sensitive to detect comparable elevations based on pooled statistics of included studies [16].

The significant effect size of the binding potential described in this meta-analysis was small and may have been influenced by publication bias, inter-study heterogeneity, or by comparing anti-psychotic naïve patients (i.e., those who have not yet taken antipsychotics) with patients actively taking antipsychotics, which has been documented to influence microglial activation [17]. Much of the interstudy heterogeneity results from poorly aligned definitions. For example, using different criteria for chronic psychosis or being at high-risk for psychosis among studies erodes confidence in the small effect size described by the authors. The authors also note that adjusting for missing data within the group examining binding potential caused the results to become non-significant, further suggesting that the binding potential may only be a weak indicator of psychosis.

Investigations of more specific populations with less heterogeneity have better highlighted a role for TSPOPET. One recent study examined the association between $\left[{ }^{18} \mathrm{~F}\right]$-FEPPA tracer uptake and structural abnormalities in patients with psychosis to observe significant positive associations between TSPO volume of distribution and morphological abnormalities in the left hippocampus [18]. The same group also examined total volume of distribution of a TSPO-PET ligand in individuals at high-risk for psychosis and found no significant difference between highrisk individuals and healthy controls in the hippocampus or dorsolateral prefrontal cortex [19], suggesting TSPO imaging may not be as useful for individuals at high-risk of developing psychosis. However, it could also suggest that the clinical classification system defining who is high and low risk could be flawed, but substantial discrepancies in the binding abilities of TSPO PET suggests that tracers are the issue, not the classification system. Others examining multiple tracers have also found that TSPOPET total volume of distribution is significantly lower in patients with first-episode psychosis or schizophrenia compared to healthy controls but not in those at high-risk $[20,21]$. However, the discrepancies of effect among different TSPO tracers is still not well understood and is thus a significant barrier to reliable use as a diagnostic or monitoring biomarker.

Sustained confidence about the link between neuroinflammation and psychosis as well as the observed inadequacies of first-generation TSPO ligands have led to further studies. A meta-review found that many first-generation TSPO-PET ligands bind to TSPO non-specifically in vivo [22]. Further, they are hindered by the need to be accompanied by genotypic testing, as some genotypes inhibit binding of the tracer in vivo [23]. Specifically, a cytosine/thymine $(\mathrm{C} / \mathrm{T})$ base pair substitution at rs6971 significantly influences radiotracer binding potential. High-affinity binders $(\mathrm{C} / \mathrm{C})$ have the greatest tracer affinity, low-affinity binders (T/T) have a 50-fold reduction in affinity, and mixed-affinity binders $(\mathrm{C} / \mathrm{T})$ express both the high-affinity and low-affinity binder TSPO in approximately equal proportion. Further, polymorphisms in the rs6971 gene coding for TSPO in humans has been shown to be related to altered tracer binding characteristics for the next generation TSPO tracers. Nonetheless, TSPO-PET imaging with second generation ligands still offers greater potential to diagnose individuals with psychosis (Table 1).

Several limitations of TSPO-PET imaging are relevant to discuss. Currently, no randomized studies exist that evaluate TSPO-PET's ability to identify individuals with psychosis, and existing nonrandomized studies are vulnerable to substantial levels of confirmation and selection bias. Further, other targets of hyperinflammatory neuroimmune responses exist and may provide superior PET imaging performance than TSPO ligands (e.g., COX1 and 2 and colony stimulating factor 1) [24]. Others exist but are outside the scope of this review. A discussion of them can be found in the cited text [25].

Importantly, two randomized controlled trials evaluating drugs that target microglial activation suggest that microglia likely do not play a causative role in schizophrenia [26, 27]. However, it is possible that neuroinflammatory therapies could still benefit some individuals with schizophrenia who have particularly high levels of neuroinflammation. This hypothesis has been investigated by examining the presence of $\mathrm{T}$ and $\mathrm{B}$ lymphocytes amongst subpopulations of schizophrenic patients, finding that $15-37 \%$ of patients with schizophrenia had elevated lymphocyte counts throughout the brain [28]. If there is a definable subpopulation of patients with schizophrenia for whom inflammation is a key driver of pathogenesis, PET imaging of microglial activation might have utility as a predictive biomarker of treatment response and a monitoring biomarker of neuroimmune functioning [10]. 
Table 1 PET tracers currently in use for the study of psychosis

\begin{tabular}{|c|c|c|c|}
\hline Tracer & Structure & $\begin{array}{l}\text { Binding Affinity } \\
\end{array}$ & In vivo Target \\
\hline \multicolumn{4}{|c|}{$\begin{array}{l}\text { Structure } \\
\text { Dopaminergic Imaging } \\
\end{array}$} \\
\hline$\left[{ }^{18} \mathrm{~F}\right] \mathrm{FDOPA}$ & & $\begin{array}{c}0.05-0.25^{\mathrm{b}} \\
\text { (depends on cortical structure) }\end{array}$ & $\begin{array}{l}\text { Substrate for amino } \\
\text { acid decarboxylase; } \\
\text { used to measure } \\
\text { dopaminergic } \\
\text { functioning in the brain }\end{array}$ \\
\hline$\left[{ }^{[8} \mathrm{F}\right] \mathrm{ASEM}$ & & $0.84 \mathrm{nM}^{\mathrm{a}}$ & $\begin{array}{l}\text { Binds to the } \alpha 7-\mathrm{nAChR} \\
\text { receptor, which is } \\
\text { expressed highly in the } \\
\text { hippocampus and is } \\
\text { thought to drive } \\
\text { downstream striatal } \\
\text { dopaminergic function }\end{array}$ \\
\hline$\left[{ }^{18} \mathrm{~F}\right] \mathrm{DBT}-10$ & & $0.6 \mathrm{nM}^{\mathrm{a}}$ & $\begin{array}{l}\text { Binds to the } \alpha 7-\mathrm{nAChR} \\
\text { receptor, which is } \\
\text { expressed highly in the } \\
\text { hippocampus and is } \\
\text { thought to drive } \\
\text { downstream striatal } \\
\text { dopaminergic function }\end{array}$ \\
\hline$\left[{ }^{[1} \mathrm{C}\right]$ Raclopride & & $1 \mathrm{nM}$ & $\begin{array}{c}\text { Dopamine } \mathrm{D}_{2} \text { receptor } \\
\text { antagonist }\end{array}$ \\
\hline \multicolumn{4}{|c|}{ Neuroimmune TSPO Imaging } \\
\hline$\left[{ }^{11} \mathrm{C}\right] \mathrm{DAA} 1106$ & & $0.2-13.1 \mathrm{nM}$ & $\begin{array}{l}\text { Binds to peripheral } \\
\text { benzodiazepine } \\
\text { receptors (TSPO) in the } \\
\text { brain }\end{array}$ \\
\hline$\left[{ }^{11} \mathrm{C}\right] \mathrm{PBR} 28$ & & $2.2-52 \mathrm{nM}$ & $\begin{array}{l}\text { Binds to peripheral } \\
\text { benzodiazepine } \\
\text { receptors (TSPO) in the } \\
\text { brain }\end{array}$ \\
\hline$\left[{ }^{11} \mathrm{C}\right]$ Ro 5-4864 & & $>40 \mathrm{nM}$ & $\begin{array}{l}\text { Binds to peripheral } \\
\text { benzodiazepine } \\
\text { receptors (TSPO) in the } \\
\text { brain }\end{array}$ \\
\hline$\left[{ }^{11} \mathrm{C}\right](R)-\mathrm{PK} 11195$ & & $2.1-28.5 \mathrm{nM}$ & $\begin{array}{l}\text { Binds to peripheral } \\
\text { benzodiazepine } \\
\text { receptors (TSPO) in the } \\
\text { brain }\end{array}$ \\
\hline$\left[{ }^{11} \mathrm{C}\right] \mathrm{PBR} 01$ & & $1.40 \mathrm{nM}$ & $\begin{array}{l}\text { Binds to peripheral } \\
\text { benzodiazepine } \\
\text { receptors (TSPO) in the } \\
\text { brain }\end{array}$ \\
\hline$\left[{ }^{13} \mathrm{C}\right] \mathrm{DPA}-713$ & & $15.0-66.4 \mathrm{nM}$ & $\begin{array}{l}\text { Binds to peripheral } \\
\text { benzodiazepine } \\
\text { receptors (TSPO) in the } \\
\text { brain }\end{array}$ \\
\hline$\left.\left.\right|^{18} \mathrm{~F}\right] \mathrm{GE}-180$ & & $1: 5-1: 15^{\mathrm{c}}$ & $\begin{array}{l}\text { Binds to peripheral } \\
\text { benzodiazepine } \\
\text { receptors (TSPO) in the } \\
\text { brain }\end{array}$ \\
\hline
\end{tabular}


Table 1 (continued)

\author{
Note: the tracers reported under the TSPO imaging section are only meant to be a sampling. More tracers \\ exist and can be found in Refs. [48, 49]. Binding affinity information for TSPO tracers in this table was \\ adapted from [48]

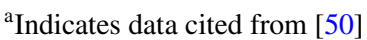 \\ ${ }^{\mathrm{b}}$ Indicates a $K_{i}$ value \\ ${ }^{\mathrm{c}}$ Indicates a ratio of high affinity genotype binding affinity to low affinity genotype binding affinity
}

\section{Dopaminergic dysfunction}

$\left[{ }^{18} \mathrm{~F}\right]$ fluoro-L-dihydroxyphenylalanine $\left(\left[{ }^{18} \mathrm{~F}\right] \mathrm{FDOPA}\right) \mathrm{PET}$ imaging of dopaminergic activity is one of the most studied diagnostic biomarkers for psychosis. The present understanding of psychosis includes dopaminergic dysfunction as a key causative factor, and all current antipsychotics target dopaminergic functioning in various ways [29]. However, different hypotheses of dopaminergic dysfunction have been suggested (e.g., presynaptic striatal dysfunction, dopamine receptor dysfunction, etc.) and are still being evaluated for their utility in the understanding of psychosis [30].

A meta-analysis of 113 patients with schizophrenia and 131 healthy controls examining presynaptic striatal dopamine synthesis capacity (DSC) measured via PET imaging found that patients with schizophrenia exhibited significantly higher levels (14\%) of DSC compared to healthy controls in the caudate nucleus and the putamen [31]. Possessing less heterogeneity than other imaging meta-analyses, this study provides strong support for elevated DSC as a diagnostic biomarker for schizophrenia. In addition, a second metaanalysis examining the density of the dopamine active transporter (DAT) in the striatum of patients with schizophrenia and healthy controls found no significant difference between patients and controls, suggesting that an abnormal density striatal dopamine receptors is not a marker for schizophrenia [32]. In another more recent study using Raclopride PET, the authors found no significant correlation between DSC and $\mathrm{D} 2$ receptor availability in the striatum but did find a significant correlation in the putamen, confirming the results above [33].

A larger meta-analysis examining 618 patients with schizophrenia and 606 healthy controls examined in vivo striatal dopaminergic function via either PET or single-photon emission computed tomography (SPECT) imaging. The authors found that there was a significant elevation in presynaptic dopaminergic levels, no change in DAT levels, and a small increase in dopamine D2/3 receptor availability [34], which is known to be associated with elevated DSC [35]. Finally, one recent study examining dopamine release during cognitive tasks among patients with schizophrenia found significant dopamine depletion when performing cognitive tasks amongst this population compared to healthy controls [36], perhaps helping explain the increase in D2/3 receptor availability found elsewhere. While these studies come to similar conclusions, it is possible that the meta-analyses were influenced by publication bias (the authors noted asymmetry in funnel plots for studies examining presynaptic dopaminergic function), non-randomized design of included studies, or confounders such as alcohol use, which is also associated with dopaminergic dysfunction [37].

Despite strong evidence for the use of dopaminergic dysfunction as a diagnostic biomarker of psychosis, limitations remain. Most individual studies are substantially limited in that they do not longitudinally assess dopaminergic dysfunction within individuals, nor do they evaluate the ability to distinguish between different psychiatric disorders involving psychosis among a randomized, blinded cohort. Regardless, existing studies show with great confidence that psychosis and high-risk status for psychosis are associated with striatal dopaminergic dysfunction, which is useful for diagnostic purposes, but cross-sectional study designs limit causal inference.

\section{a7-nAChR receptor}

Elevated dopaminergic activity in the associative striatum has led to the search for upstream targets that could be modulated to quell downstream dysfunction. Recent studies have found that hippocampal hyperactivity may drive dopaminergic dysfunction in the striatum [10,38]. Accordingly, efforts have targeted the $\alpha 7$ nicotinic cholinergic receptor $(\alpha 7$-nAChR), which is expressed on GABAergic interneurons in the hippocampus [39]. Existing research suggests that the density of the $\alpha 7-\mathrm{nAChR}$ is lower in the hippocampi of people with schizophrenia than healthy controls [40] and that abnormalities in the gene encoding the receptor are present at a higher rate in schizophrenics than healthy controls [41], making it a promising therapeutic target for those with psychosis and a potential diagnostic biomarker (Fig. 1).

Limited research has been conducted with $\left[{ }^{18} \mathrm{~F}\right]$-ASEM (3-(1,4-diazabicyclo[3.2.2]nonan-4-yl)-6-18Ffluorodibenzo[b,d]thiophene 5,5 -dioxide $\left(\left[{ }^{18} \mathrm{~F}\right] \mathrm{ASEM}\right)$ PET imaging of the $\alpha 7$-nAChR in psychosis. In one crosssectional study of 11 patients with recent-onset psychosis and 15 healthy controls, researchers used $\left[{ }^{18} \mathrm{~F}\right]$ ASEM PET to measure the availability of the $\alpha 7-n A C h R$ in the hippocampi [42]; the study found that those with non-affective psychosis, had significantly lower binding of the $\left[{ }^{18} \mathrm{~F}\right]$ ASEM PET ligands in the hippocampi compared to controls, 


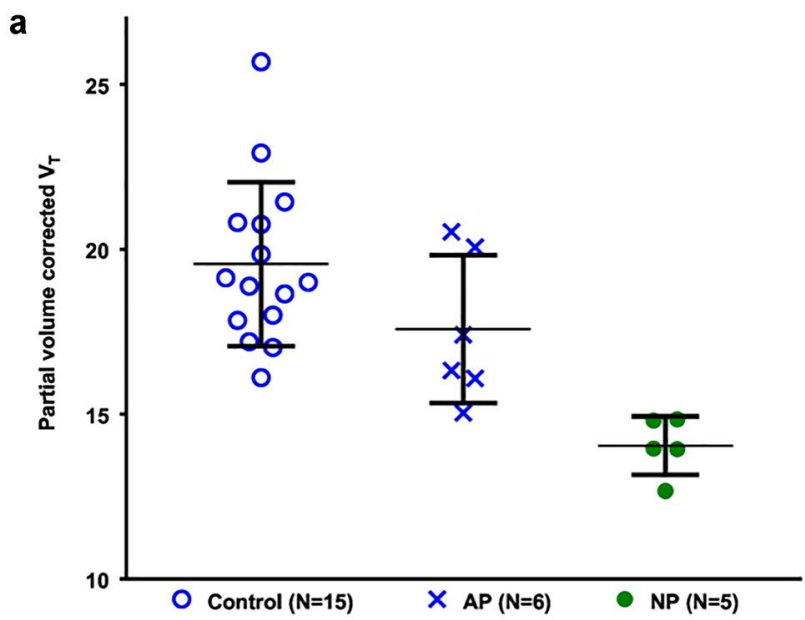

b

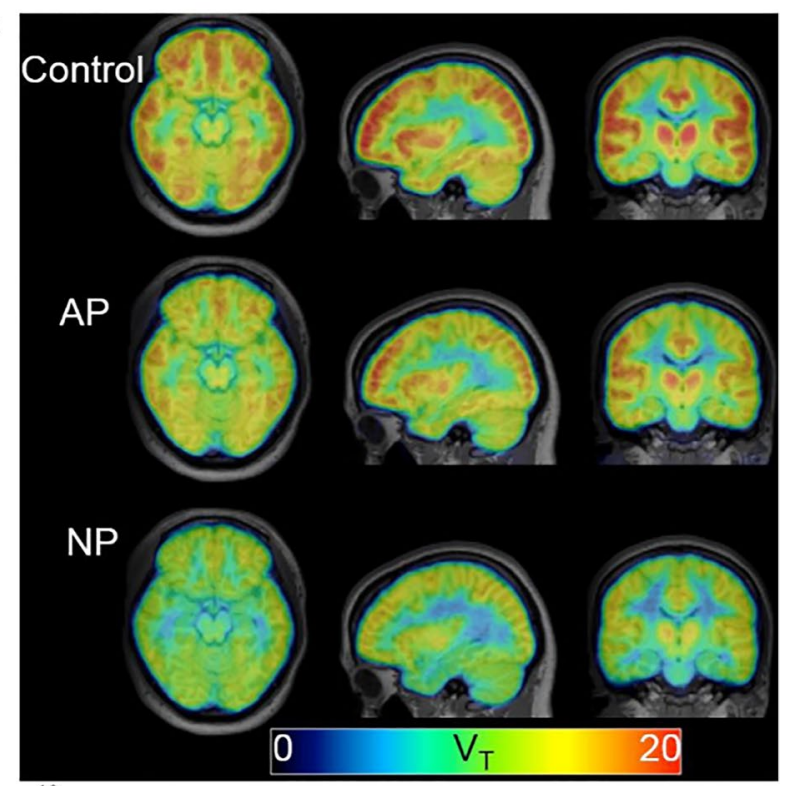

Fig. 1 "Comparison between ${ }^{18} \mathrm{~F}$-ASEM total distribution volume $\left(V_{T}\right)$ values from non-smoking participants that were grouped as healthy controls $(N=15)$, patients with recent-onset of affective psychosis (AP) $(N=6)$ or patients with recent-onset of non-affective psychosis (NP) $(N=5)$. a Scatterplot of ${ }^{18} \mathrm{~F}$-ASEM $V_{T}$ values in hippocampus from healthy controls, patients with AP and patients with NP. $V_{T}$ was estimated from images corrected for partial volume effects and mean and standard deviation values are shown (lines). b Mean parametric ${ }^{18} \mathrm{~F}$-ASEM $V_{T}$ images derived from PET data that were uncorrected for partial volume effects from the study population of 15 controls (top panel), six patients with AP (middle panel), and five patients with NP (lower panel) suggest group differences in binding outside the hippocampus as well. Images are displayed in groups of three views (left to right: axial, sagittal, coronal) and $V_{T}$ is in units of $\mathrm{mL} \mathrm{cm}^{-3}$." This figure was reproduced with permission from [42]."

underscoring this modality as a potential diagnostic tool. Imaging studies in both animals and humans have confirmed that binding of the ASEM PET ligand was lower in controls than in subjects with psychosis [43-45]. However, our understanding of the $\alpha 7$-nAChR in patients with psychosis is still preliminary. Further, to the author's knowledge, no prospective, blinded studies have been conducted evaluating $\left[{ }^{18}\right.$ F]ASEM PET's accuracy in identifying patients with psychosis over healthy controls. Additionally, recent studies suggest that levels of the $\alpha 7-n A C h R$ are abnormal in individuals with mild cognitive impairment [46] and change naturally with aging [47], both of which would confound the tracer's ability to identify individuals with psychosis over other neuropathological illnesses. Used alongside professional judgement and symptomatic evaluation, however, $\left[{ }^{18} \mathrm{~F}\right]$ ASEM PET may possess utility as a diagnostic marker of psychosis.

\section{Outcome biomarkers}

\section{Prognostic biomarkers}

PET imaging prognostic biomarkers have centred around the use of dopaminergic dysfunction in patients at high-risk for developing psychosis. A study by Howes et al. examined longitudinal, within-subject changes in striatal DSC among individuals who transitioned from high-risk to psychotic states [51]. Twenty subjects were scanned with $\left[{ }^{18} \mathrm{~F}\right] \mathrm{FDOPA}$ PET on two separate occasions: first when presenting as high-risk patients and then again 2 years later. At the second scan, eight subjects had developed a psychotic disorder. This study showed that there was a significant increase in the rate constant $\left(k_{i}^{\text {cer }}\right)$ of subjects who developed psychosis between the baseline and final scan in the associative striatum only, with the greatest difference being in the right putamen [51] (Fig. 2).

At the time of publishing, this study did not offer conclusive evidence that striatal DSC could be used as a prognostic biomarker in high-risk individuals, mainly because the data did not show that the increase in the transition group was different from any increases in the group that was high-risk but did not develop psychosis. This suggests that either elevated DSC may not be specific to the development of psychosis or that the effects are due to a small sample size. The authors subsequently published a study on a larger cohort that found significant differences between the transition group (from high-risk to psychosis) and the non-transition group, as well as between the transition group and healthy controls, suggesting that the original limitations of the study were due to sample size [52]. Taken together, these results suggest that DSC (measured via $k_{i}^{\text {cer }}$ ) in patients at high-risk for developing psychosis can be used as a prognostic biomarker to identify those who will develop schizophrenia, although additional research is needed in this area. 


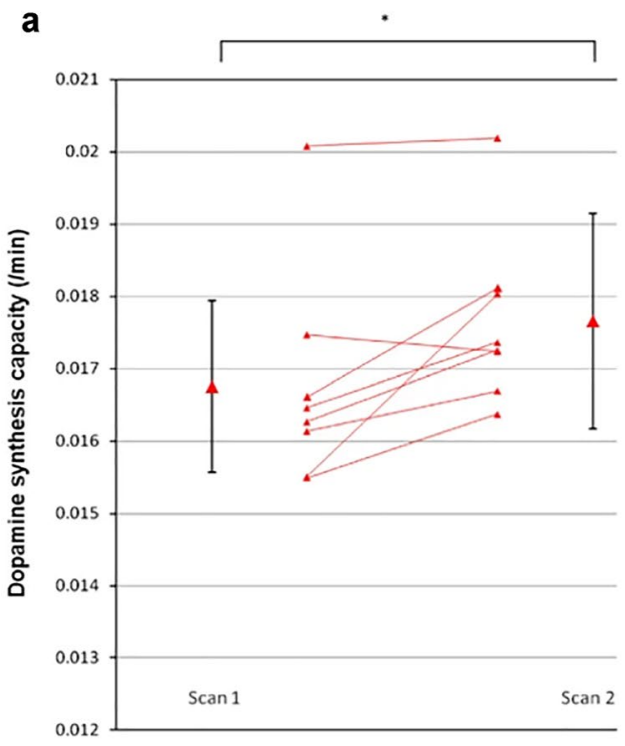

Fig. 2 a "Showing within-subject changes in striatal dopamine synthesis capacity in the sensorimotor striatum of subjects who had developed psychosis by scan 2 . The group mean (error bars $=\mathrm{sd}$ ) is shown at the side for each time point $\left({ }^{*} P<0.05\right)$." $\mathbf{b}$ "Progressive increase in dopamine synthesis capacity in subjects who made the

\section{Predictive biomarkers}

Approximately one third of patients with schizophrenia are treatment resistant [8]. Identification of patients who will respond to antipsychotic therapy will thus hasten the application of effective treatments and avoid unnecessary physical, social, and monetary costs associated with taking ineffective treatments.

The percent occupancy of dopamine $\mathrm{D} 2$ receptors has been studied for its ability to predict one's response to antipsychotic therapies. In one study, researchers used SPECT to measure in vivo occupancy of striatal D2 receptors in 18 healthy controls and 18 treatment naïve schizophrenics. The study determined that intrasynaptic dopamine depletion resulted in significantly less D2 receptor occupancy in patients with schizophrenia than in healthy controls [53]. Further, schizophrenia patients with elevated synaptic dopamine levels experience greater responses to antipsychotics than those with normal synaptic dopamine levels [53]. Importantly, the predictive capabilities only held for the reduction of positive symptoms, as there was no significant change in negative symptoms across the cohort [53]. Although D2 receptor occupancy and synaptic dopamine levels were measured via SPECT in this work, this study offered a promising proof-of-concept for subsequent PET imaging studies.

PET studies have produced comparable results. In a retrospective study comparing 12 treatment-responsive with 12 treatment-nonresponsive patients with Schizophrenia and 12 b
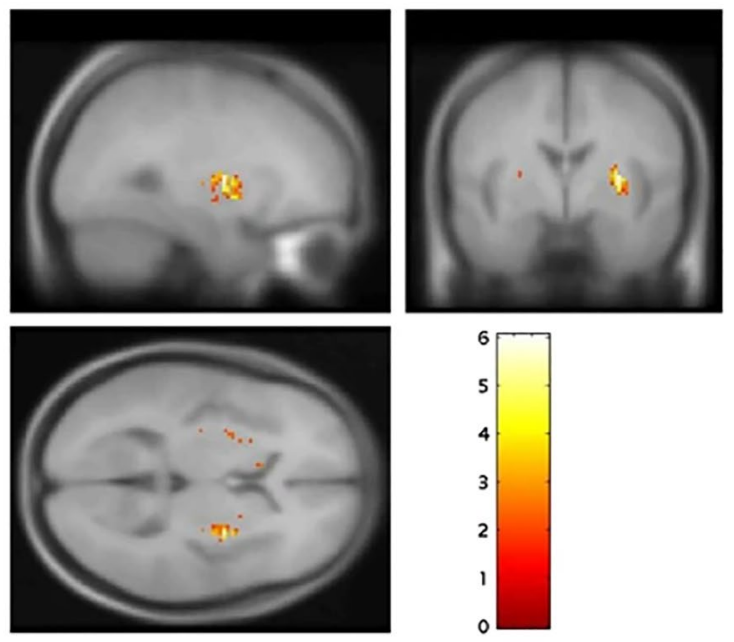

transition from the prodromal phase to a first episode of psychosis. There was a longitudinal increase in the right putamen (cluster extent: 124 voxels, MNI coordinates of peak voxel $30,-4,6 ; P=0.008$ cluster corrected)." These figures were reproduced and combined into one figure with permission from [51]

healthy controls, researchers found that $\left[{ }^{18} \mathrm{~F}\right] \mathrm{FDOPA}$ uptake, measured by $k_{i}^{\text {cer }}$, was greater in the associative and limbic striatum of the treatment-responsive group when compared to both the treatment-resistant and healthy control groups. Notably, there was no difference between the treatmentnonresponsive group and healthy controls, indicating that this predictive marker could have a low specificity (Fig. 3). A subsequent prospective study of 26 antipsychotic-naïve patients with first-episode psychosis and 14 healthy controls examined $\left[{ }^{18} \mathrm{~F}\right]$ FDOPA uptake and revealed that the $k_{i}^{\text {cer }}$ in the associative striatum was significantly higher in patients who demonstrated a positive response to antipsychotics than those who did not [54], underscoring differences in baseline striatal dopaminergic function detectable at the first episode of psychosis.

Current work suggests that $\left[{ }^{18} \mathrm{~F}\right] \mathrm{FDOPA}$ PET is a reliable marker of treatment response but not of treatment nonresponse. A recent study assessing DSC in six antipsychotic non-responders with psychosis, eight responders, and ten healthy controls found that DSC levels were significantly higher in responders than healthy controls, confirming the positive predictive capability of DSC for psychosis [55]. However, the researchers noted that glutamate levels were significantly higher in the anterior cingulate cortex in treatment non-responders compared to healthy controls [55]. This has been noted in other cohorts as well [56, 57], suggesting that glutamate levels (measured via proton magnetic resonance spectroscopy) alongside $\left[{ }^{18} \mathrm{~F}\right]$ FDOPA PET may represent the ideal way to reliably predict treatment response 

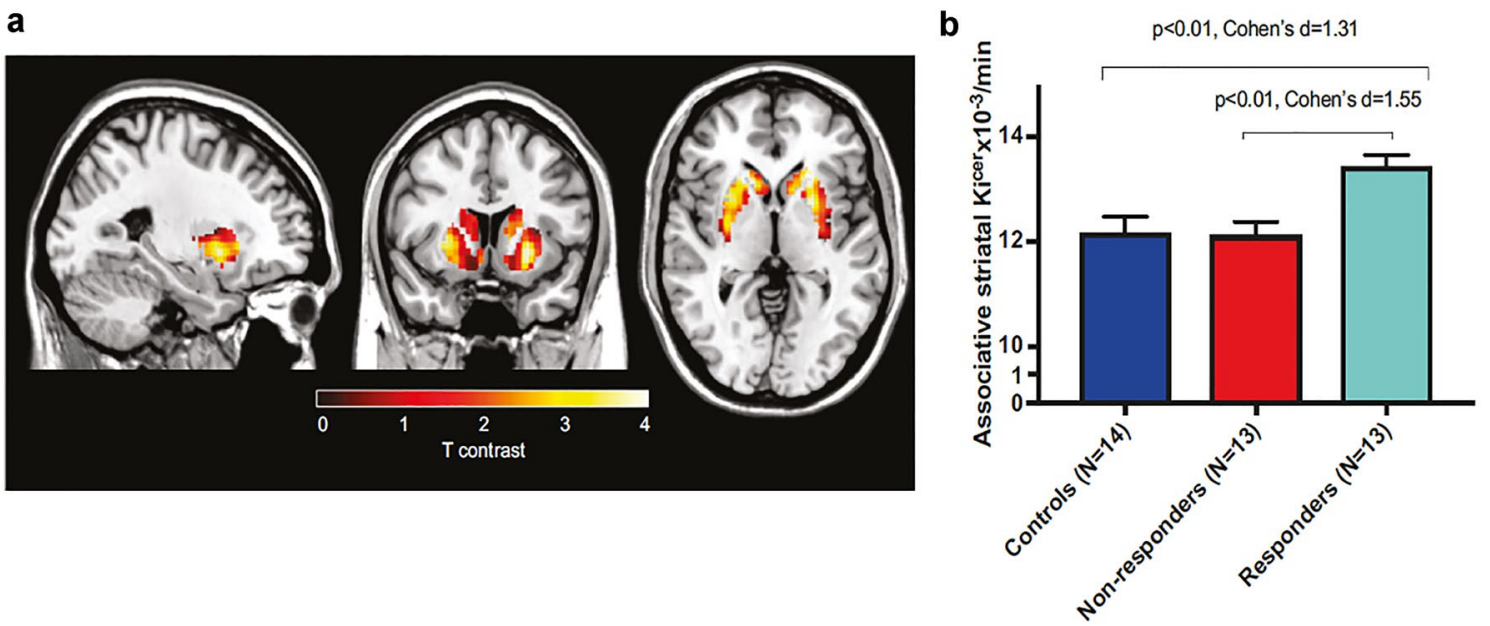

Fig. 3 a "Voxel-wise analysis of treatment responders compared to non-responders. Increased dopamine synthesis capacity, relative to non-responder patients $(N=13)$, in patients who respond to treatment $(N=13)$. The most significant increase was in voxels in right caudate (peak MNI coordinates $x=18, y=20, z=2$; pFWE corr $=0.026$ ) and left putamen (peak MNI coordinates $x=-24, y=8, z=-2$; pFWE

and non-response among individuals with psychosis. However, the concurrent use of these two modalities is resourceintensive, which further highlights the need for a unified predictive PET marker of psychosis.

Additional efforts have been made to bypass the need to use two biomarkers to predict response. For instance, some recent work has paired $\left[{ }^{18} \mathrm{~F}\right]$ FDOPA PET with machine learning methods of analysis to identify potential responders, achieving $40-60 \%$ sensitivity at $100 \%$ specificity [58]. Others have combined measurements of DSC via $\left[{ }^{18} \mathrm{~F}\right]$ FDOPA PET with measures of functional connectivity in the frontostriatal circuits measured via fMRI [59]. This work reliably identified the individuals who had a positive response to first-line antipsychotics [59]. However, these methods are largely new and relatively understudied such that they require additional research to better determine efficacy.

\section{Conclusion}

PET imaging biomarkers have great promise as diagnostic, prognostic, and predictive markers for psychosis. At present, biomarkers with the greatest possible clinical utility have centred around measuring neuroinflammation, dopaminergic dysfunction, and the $\alpha 7-n A C h R$ receptor density. Existing research is limited by a lack of randomized controlled trials evaluating the sensitivity and specificity of such markers in diagnosing and predicting psychosis and treatment response. Further, the ability of existing PET ligands to detect psychosis over other illnesses (and subtypes of psychosis) is limited corr=0.027)." b "Mean dopamine synthesis capacity by group. Dopamine synthesis capacity is elevated at baseline in patients who subsequently respond to antipsychotic treatment, compared to controls and nonresponders (Cohen's $d$ effect size $=1.31$ and 1.55, respectively). Error bars indicate standard error of the mean.” Figures reproduced with permission without changes from [54]

by a lack of randomized, prospective trails and suggests that PET tracers offer the most utility when used in conjunction with a clinical evaluation. Nonetheless, PET imaging is an evolving and promising means of improving the diagnosis and management of psychosis.

Author contributions GR: design of study, research, writing, editing; CA: writing, editing; TW: writing, editing, study oversight; AA: design of study, editing, oversight of study; MER: writing, editing, oversight of study.

Funding Open access funding provided by University of Oslo (incl Oslo University Hospital). GR was funded by the Rhodes Trust during the completion of this project, but the Rhodes Trust had no role in any part of this study.

Data availability All data used in this article is available in the included articles.

Code availability Not applicable.

\section{Declarations}

Conflict of interest The authors have no relevant financial or non-financial conflict of interest to declare.

Open Access This article is licensed under a Creative Commons Attribution 4.0 International License, which permits use, sharing, adaptation, distribution and reproduction in any medium or format, as long as you give appropriate credit to the original author(s) and the source, provide a link to the Creative Commons licence, and indicate if changes were made. The images or other third party material in this article are included in the article's Creative Commons licence, unless indicated otherwise in a credit line to the material. If material is not included in 
the article's Creative Commons licence and your intended use is not permitted by statutory regulation or exceeds the permitted use, you will need to obtain permission directly from the copyright holder. To view a copy of this licence, visit http://creativecommons.org/licenses/by/4.0/.

\section{References}

1. Biomarkers Definitions Working Group (2001) Biomarkers and surrogate endpoints: preferred definitions and conceptual framework. Clin Pharmacol Ther 69:89-95

2. BEST (Biomarkers, Endpoints, and other Tools) Resource F-NBW (2016) Understanding prognostic versus predictive biomarkers [internet]. BEST Biomark. EndpointS Tools Resour. Internet. Food and Drug Administration (US). https://www.ncbi.nlm.nih. gov/books/NBK402284/. Accessed 28 Apr 2021

3. Gaebel W, Zielasek J (2015) Focus on psychosis. Dialogues Clin Neurosci 17:9-18

4. Arciniegas DB (2015) Psychosis. Contin Lifelong Learn Neurol 21:715-736

5. Jongsma HE, Turner C, Kirkbride JB, Jones PB (2019) International incidence of psychotic disorders, 2002-17: a systematic review and meta-analysis. Lancet Public Health 4:e229-e244

6. Baca-Garcia E, Perez-Rodriguez MM, Basurte-Villamor I, Moral ALFD, Jimenez-Arriero MA, Rivera JLGD et al (2007) Diagnostic stability of psychiatric disorders in clinical practice. Br J Psychiatry 190:210-216

7. Kapur S, Phillips AG, Insel TR (2012) Why has it taken so long for biological psychiatry to develop clinical tests and what to do about it? Mol Psychiatry 17:1174-1179

8. Lindenmayer JP (2000) Treatment refractory schizophrenia. Psychiatr Q 71:373-384

9. Hellwig S, Domschke K (2019) Update on PET imaging biomarkers in the diagnosis of neuropsychiatric disorders. Curr Opin Neurol 32:539-547

10. Coughlin JM, Horti AG, Pomper MG (2019) Opportunities in precision psychiatry using PET neuroimaging in psychosis. Neurobiol Dis 131:104428

11. Cannon TD (2016) Microglial activation and the onset of psychosis. Am J Psychiatry 173:3-4

12. Crawshaw AA, Robertson NP (2017) The role of TSPO PET in assessing neuroinflammation. J Neurol 264:1825-1827

13. Banati R, Hickie IB (2009) Therapeutic signposts: using biomarkers to guide better treatment of schizophrenia and other psychotic disorders. Med J Aust 190. https://onlinelibrary.wiley. com/doi/abs/10.5694/j.1326-5377.2009.tb02371.x. Accessed 12 May 2021

14. Doorduin J, de Vries EFJ, Willemsen ATM, de Groot JC, Dierckx RA, Klein HC (2009) Neuroinflammation in schizophrenia-related psychosis: a PET study. J Nucl Med 50:1801-1807

15. Bloomfield PS, Selvaraj S, Veronese M, Rizzo G, Bertoldo A, Owen DR et al (2015) Microglial activity in people at ultra high risk of psychosis and in schizophrenia: an [11C]PBR28 PET brain imaging study. Am J Psychiatry 173:44-52

16. Marques TR, Ashok AH, Pillinger T, Veronese M, Turkheimer FE, Dazzan P et al (2019) Neuroinflammation in schizophrenia: meta-analysis of in vivo microglial imaging studies. Psychol Med 49:2186-2196

17. Holmes SE, Hinz R, Drake RJ, Gregory CJ, Conen S, Matthews JC et al (2016) In vivo imaging of brain microglial activity in antipsychotic-free and medicated schizophrenia: a [11 C](R)PK11195 positron emission tomography study. Mol Psychiatry 21:1672-1679
18. Hafizi S, Guma E, Koppel A, Da Silva T, Kiang M, Houle S et al (2018) TSPO expression and brain structure in the psychosis spectrum. Brain Behav Immun 74:79-85

19. Hafizi S, Da Silva T, Gerritsen C, Kiang M, Bagby RM, Prce I et al (2017) Imaging microglial activation in individuals at clinical high risk for psychosis: an in vivo PET study with [18 F]FEPPA. Neuropsychopharmacology 42:2474-2481

20. Collste K, Plavén-Sigray P, Fatouros-Bergman H, Victorsson P, Schain M, Forsberg A et al (2017) Lower levels of the glial cell marker TSPO in drug-naive first-episode psychosis patients as measured using PET and [11 C] PBR28. Mol Psychiatry 22:850-856

21. Plavén-Sigray P, Matheson GJ, Collste K, Ashok AH, Coughlin JM, Howes OD et al (2018) Positron emission tomography studies of the glial cell marker translocator protein in patients with psychosis: a meta-analysis using individual participant data. Biol Psychiatry 84:433-442

22. De Picker LJ, Morrens M, Chance SA, Boche D (2017) Microglia and brain plasticity in acute psychosis and schizophrenia illness course: a meta-review. Front Psychiatry 8:238

23. Mizrahi R, Rusjan PM, Kennedy J, Pollock B, Mulsant B, Suridjan I et al (2012) Translocator protein (18 kDa) polymorphism (rs6971) explains in-vivo brain binding affinity of the PET radioligand [18F]-FEPPA. J Cereb Blood Flow Metab 32:968-972

24. Prabhakaran J, Molotkov A, Mintz A, Mann JJ (2021) Progress in PET imaging of neuroinflammation targeting COX-2 enzyme. Molecules 26:3208

25. Janssen B, Vugts DJ, Windhorst AD, Mach RH (2018) PET imaging of microglial activation-beyond targeting TSPO. Molecules 23:607

26. Deakin B, Suckling J, Barnes TRE, Byrne K, Chaudhry IB, Dazzan P et al (2018) The benefit of minocycline on negative symptoms of schizophrenia in patients with recent-onset psychosis (BeneMin): a randomised, double-blind, placebo-controlled trial. Lancet Psychiatry 5:885-894

27. Weiser M, Levi L, Burshtein S, Chiriță R, Cirjaliu D, Gonen I et al (2019) The effect of minocycline on symptoms in schizophrenia: results from a randomized controlled trial. Schizophr Res 206:325-332

28. Bogerts B, Winopal D, Schwarz S, Schlaaff K, Dobrowolny $\mathrm{H}$, Mawrin C et al (2017) Evidence of neuroinflammation in subgroups of schizophrenia and mood disorder patients: a semiquantitative postmortem study of CD3 and CD20 immunoreactive lymphocytes in several brain regions. Neurol Psychiatry Brain Res 23:2-9

29. Howes OD, Kapur S (2014) A neurobiological hypothesis for the classification of schizophrenia: type a (hyperdopaminergic) and type B (normodopaminergic). Br J Psychiatry 205:1-3

30. Grace AA (2000) Gating of information flow within the limbic system and the pathophysiology of schizophrenia. Brain Res Rev 31:330-341

31. Fusar-Poli P, Meyer-Lindenberg A (2013) Striatal presynaptic dopamine in schizophrenia, part II: meta-analysis of [(18)F/(11) C]-DOPA PET studies. Schizophr Bull 39:33-42

32. Fusar-Poli P, Meyer-Lindenberg A (2013) Striatal presynaptic dopamine in schizophrenia, part I: meta-analysis of dopamine active transporter (DAT) density. Schizophr Bull 39:22-32

33. Yamamoto Y, Takahata K, Kubota M, Takano H, Takeuchi H, Kimura Y et al (2021) Differential associations of dopamine synthesis capacity with the dopamine transporter and D2 receptor availability as assessed by PET in the living human brain. Neuroimage 226:117543

34. Howes OD, Kambeitz J, Kim E, Stahl D, Slifstein M, AbiDargham A et al (2012) The nature of dopamine dysfunction in schizophrenia and what this means for treatment. Arch Gen Psychiatry 69:776-786 
35. Berry AS, Shah VD, Furman DJ, White RL III, Baker SL, O'Neil JP et al (2018) Dopamine synthesis capacity is associated with D2/3 receptor binding but not dopamine release. Neuropsychopharmacology 43:1201-1211

36. Rao N, Northoff G, Tagore A, Rusjan P, Kenk M, Wilson A et al (2019) Impaired prefrontal cortical dopamine release in schizophrenia during a cognitive task: a [11C]FLB 457 positron emission tomography study. Schizophr Bull 45:670-679

37. Heinz A, Siessmeier T, Wrase J, Hermann D, Klein S, GrüsserSinopoli SM et al (2004) Correlation between dopamine D2 receptors in the ventral striatum and central processing of alcohol cues and craving. Am J Psychiatry 161:1783-1789

38. Grace AA, Gomes FV (2019) The circuitry of dopamine system regulation and its disruption in schizophrenia: insights into treatment and prevention. Schizophr Bull 45:148-157

39. Neves GA, Grace AA (2018) $\alpha 7$ Nicotinic receptor-modulating agents reverse the hyperdopaminergic tone in the MAM model of schizophrenia. Neuropsychopharmacology 43:1712-1720

40. Schaaf CP (2014) Nicotinic acetylcholine receptors in human genetic disease. Genet Med 16:649-656

41. Leonard S, Gault J, Hopkins J, Logel J, Vianzon R, Short M et al (2002) Association of promoter variants in the $\alpha 7$ nicotinic acetylcholine receptor subunit gene with an inhibitory deficit found in schizophrenia. Arch Gen Psychiatry 59:1085-1096

42. Coughlin JM, Du Y, Crawford JL, Rubin LH, Azad BB, Lesniak WG et al (2019) Use of 18F-ASEM PET to determine the availability of the $\alpha 7$-nicotinic acetylcholine receptor in recent-onset psychosis. J Nucl Med 60:241-243

43. Horti AG, Gao Y, Kuwabara H, Wang Y, Abazyan S, Yasuda $\mathrm{RP}$ et al (2014) 18F-ASEM, a radiolabeled antagonist for imaging the $\alpha 7$-nicotinic acetylcholine receptor with PET. J Nucl Med 55:672-677

44. Wong DF, Kuwabara H, Pomper M, Holt DP, Brasic JR, George $\mathrm{N}$ et al (2014) Human brain imaging of $\alpha 7 \mathrm{nAChR}$ with [18F] ASEM: a new PET radiotracer for neuropsychiatry and determination of drug occupancy. Mol Imaging Biol 16:730-738

45. Wong DF, Kuwabara H, Horti AG, Roberts JM, Nandi A, Cascella $\mathrm{N}$ et al (2018) Brain PET imaging of $\alpha 7-n A C h R$ with [18F] ASEM: reproducibility, occupancy, receptor density, and changes in schizophrenia. Int J Neuropsychopharmacol 21:656-667

46. Coughlin JM, Rubin LH, Du Y, Rowe SP, Crawford JL, Rosenthal $\mathrm{HB}$ et al (2020) High availability of the $\alpha 7$-nicotinic acetylcholine receptor in brains of individuals with mild cognitive impairment: a pilot study using 18F-ASEM PET. J Nucl Med 61:423-426

47. Coughlin JM, Du Y, Rosenthal HB, Slania S, Min Koo S, Park A et al (2018) The distribution of the alpha7 nicotinic acetylcholine receptor in healthy aging: an in vivo positron emission tomography study with [18F]ASEM. Neuroimage 165:118-124

48. Alam MdM, Lee J, Lee S-Y (2017) Recent progress in the development of TSPO PET ligands for neuroinflammation imaging in neurological diseases. Nucl Med Mol Imaging 51:283-296
49. Zhang L, Hu K, Shao T, Hou L, Zhang S, Ye W et al (2021) Recent developments on PET radiotracers for TSPO and their applications in neuroimaging. Acta Pharm Sin B 11:373-393

50. Hillmer AT, Zheng M-Q, Li S, Scheunemann M, Lin S, Holden $\mathrm{D}$ et al (2016) PET imaging evaluation of [18F]DBT-10, a novel radioligand specific to $\alpha 7$ nicotinic acetylcholine receptors, in nonhuman primates. Eur J Nucl Med Mol Imaging 43:537-547

51. Howes O, Bose S, Turkheimer F, Valli I, Egerton A, Stahl D et al (2011) Progressive increase in striatal dopamine synthesis capacity as patients develop psychosis: a PET study. Mol Psychiatry $16: 885-886$

52. Howes OD, Bose SK, Turkheimer F, Valli I, Egerton A, Valmaggia LR et al (2011) Dopamine synthesis capacity before onset of psychosis: a prospective [18F]-DOPA PET imaging study. Am J Psychiatry 168:1311-1317

53. Abi-Dargham A, Rodenhiser J, Printz D, Zea-Ponce Y, Gil R, Kegeles LS et al (2000) Increased baseline occupancy of D2 receptors by dopamine in schizophrenia. Proc Natl Acad Sci 97:8104-8109

54. Jauhar S, Veronese M, Nour MM, Rogdaki M, Hathway P, Turkheimer FE et al (2019) Determinants of treatment response in first-episode psychosis: an 18 F-DOPA PET study. Mol Psychiatry 24:1502-1512

55. Demjaha A, Egerton A, Murray RM, Kapur S, Howes OD, Stone JM et al (2014) Antipsychotic treatment resistance in schizophrenia associated with elevated glutamate levels but normal dopamine function. Biol Psychiatry 75:e11-e13

56. Egerton A, Brugger S, Raffin M, Barker GJ, Lythgoe DJ, McGuire PK et al (2012) Anterior cingulate glutamate levels related to clinical status following treatment in first-episode schizophrenia. Neuropsychopharmacology 37:2515-2521

57. Mouchlianitis E, Bloomfield MAP, Law V, Beck K, Selvaraj S, Rasquinha N et al (2016) Treatment-resistant schizophrenia patients show elevated anterior cingulate cortex glutamate compared to treatment-responsive. Schizophr Bull 42:744-752

58. Veronese M, Santangelo B, Jauhar S, D’Ambrosio E, Demjaha A, Salimbeni H et al (2021) A potential biomarker for treatment stratification in psychosis: evaluation of an [18 F] FDOPA PET imaging approach. Neuropsychopharmacology 46:1122-1132

59. Kim S, Jung WH, Howes OD, Veronese M, Turkheimer FE, Lee Y-S et al (2019) Frontostriatal functional connectivity and striatal dopamine synthesis capacity in schizophrenia in terms of antipsychotic responsiveness: an [18F]DOPA PET and fMRI study. Psychol Med 49:2533-2542

Publisher's Note Springer Nature remains neutral with regard to jurisdictional claims in published maps and institutional affiliations. 